Supporting Information

\title{
CoP Nanoparticles in Situ Grown in Three-Dimensional Hierarchical Nanoporous Carbons as Superior Electrocatalysts for Hydrogen Evolution
}

\author{
Weiyong Yuan, ${ }^{* \dagger,}$ Xiaoyan Wang, ${ }^{\dagger,}$ Xiaoling Zhong, ${ }^{\dagger,}$ and Chang Ming Li ${ }^{\dagger,}$ \\ ${ }^{\dagger}$ Institute for Clean energy \& Advanced Materials, Faculty of Materials \& Energy, \\ Southwest University, Chongqing 400715, China. \\ "Chongqing Key Laboratory for Advanced Materials and Technologies of Clean Energies, \\ Chongqing 400715, China. \\ *Corresponding author. E-mail address: yuanweiyong@swu.edu.cn
}


RHE calibration. The calibration was performed in high-purity hydrogen saturated 0.5 $\mathrm{M} \mathrm{H}_{2} \mathrm{SO}_{4}$ with Pt foil as the working electrode, SCE as the reference electrode, and another piece of $\mathrm{Pt}$ foil as the counter electrode. $\mathrm{CV}$ measurement was carried out with a scan rate of $1 \mathrm{mV} / \mathrm{s}$, and the average of the two potentials at which the current cross zero is taken as the thermodynamic potential for the hydrogen electrode reaction (Fig. 1).

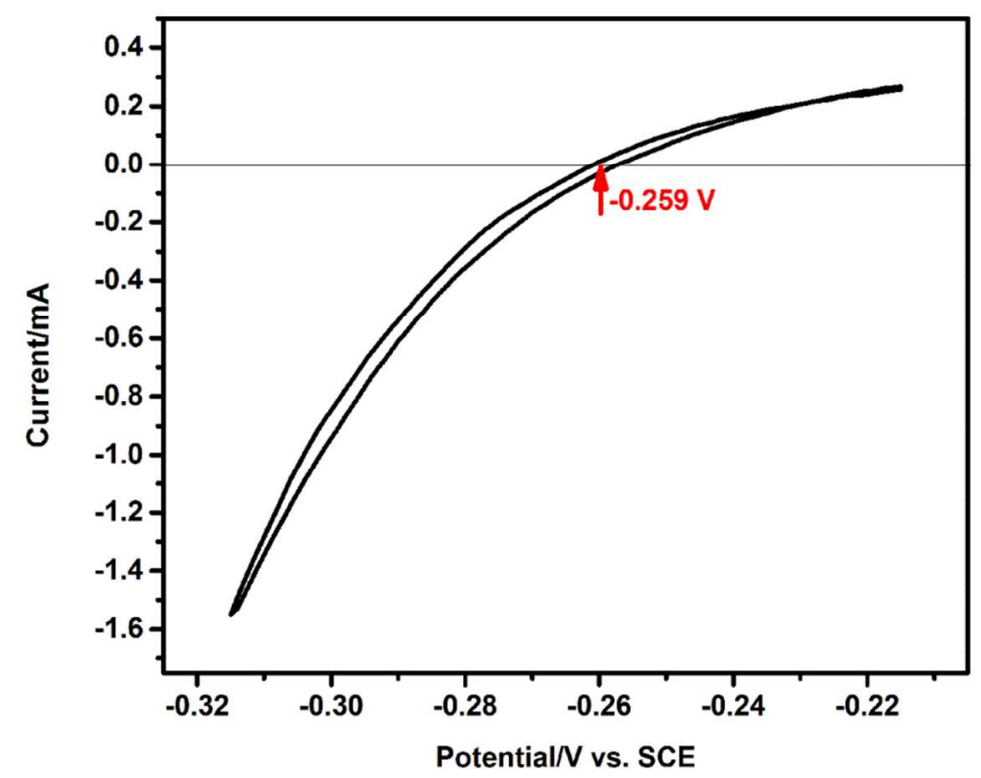

Fig. S1 Cyclic voltammogram of Pt foil in $\mathrm{H}_{2}$-saturated $0.5 \mathrm{M} \mathrm{H}_{2} \mathrm{SO}_{4}$ with a scan rate of $1 \mathrm{mV} / \mathrm{s}$. 


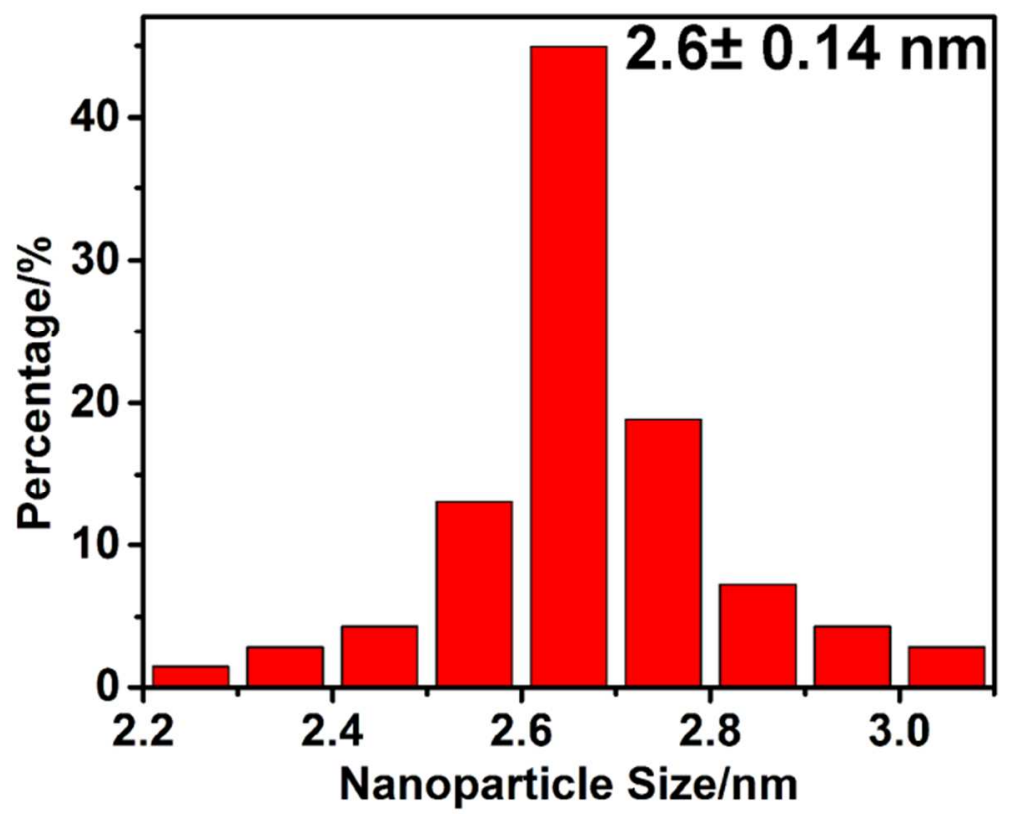

Fig. S2 Size distribution of CoP NPs in CoP/BMHNC. 

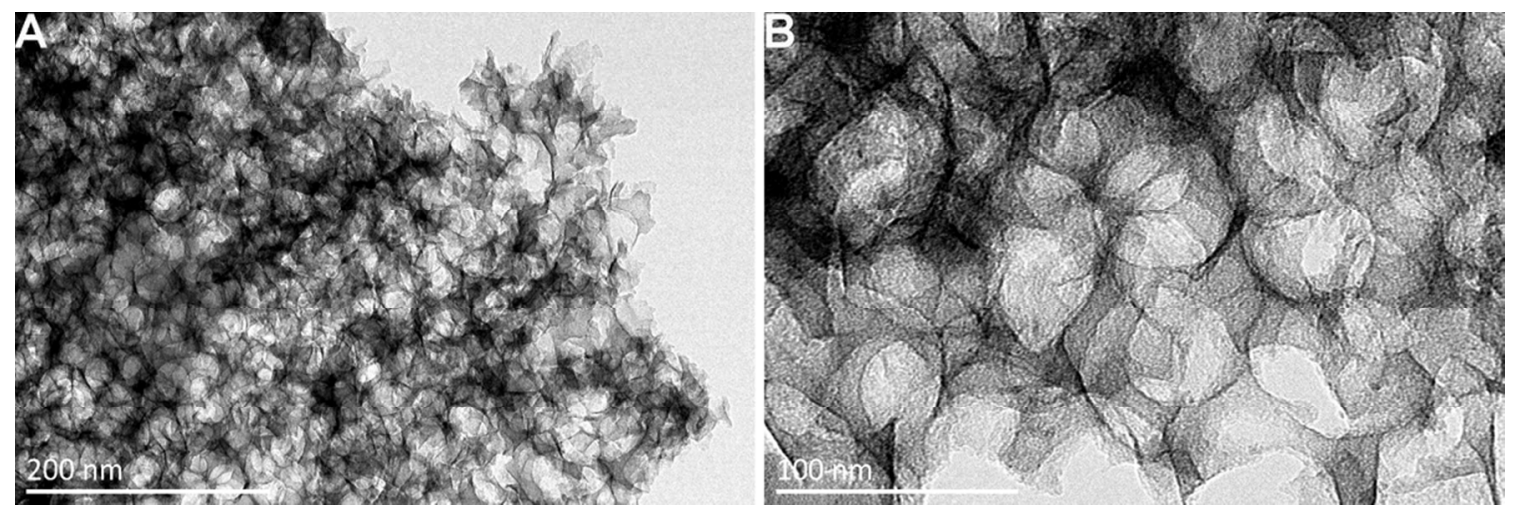

Fig. S3 TEM images of NFHNC with different magnifications. 